\title{
The real risks of steroid injection for plantar fasciitis, with a review of conservative therapies
}

\author{
Yusuf Ziya Tatli $\cdot$ Sameer Kapasi
}

Published online: 19 September 2008

(C) Humana Press 2008

\begin{abstract}
This article presents a review of conservative therapies for plantar fasciitis pain reduction with a discussion of steroid therapy risks. The therapies reviewed include orthoses, stretching, extracorporeal shockwave, BTX-A, and corticosteroid injection/iontophoresis. These modes were included based on the availability of double blinded randomized controlled trials. We noted the following findings. Orthoses, regardless of type, can improve pain levels. Plantar stretching shows limited short-term benefit (1 month), but can reflect significant long-term improvement (10 months). Extracorporeal shockwave therapy shows equivocal benefit with some studies showing significant improvement and others showing none. Although BTX-A injections were the least studied, significant pain improvement was demonstrated in the short and long term. Steroid injection/iontophoresis showed significant improvement in the short term (1 month). Steroid therapy, when coupled with plantar stretching, can provide efficacious pain relief; however, steroid injections should be combined with ultrasound monitoring to reduce complications.
\end{abstract}

Keywords Plantar fasciitis - Steroid injection Extracorporeal shockwave therapy · Stretching ·

Orthoses - BTX-A

Plantar fasciitis is a common foot disorder responsible for approximately one million physician visits per year [1]. While there are a plethora of management techniques for plantar fasciitis, there are none that are presented as a

Y. Z. Tatli $(\bowtie) \cdot$ S. Kapasi

Tufts Medical Center, 800 Washington Street, Box \#400,

Boston, MA 02111, USA

e-mail: ytatli@tuftsmedicalcenter.org; yetatli@yahoo.com definitive technique. In addition, the etiology of plantar fasciitis is not well understood [2]. Accordingly, this article will focus on a variety of conservative therapies with a follow-up focus on steroid injections and the risks thereof.

\begin{abstract}
Anatomy
The plantar fascia is synonymous with the deep fascia of the sole of the foot. The plantar fascia is comprised of pearly white longitudinally organized fibers. It begins at the medial tuberosity of the calcaneus where it is thinner and extends into a thicker center portion. This thicker portion is flanked by thinner lateral and medial portions. The thicker central portion of the plantar fascia then extends into five bands surrounding the digital tendons (Fig. 1) [2, 3].

Plantar fasciitis classically presents histologically with "degenerative changes in the plantar fascia, with or without fibroelastic proliferation and chronic inflammatory changes" [4].
\end{abstract}

\section{Mechanics of the plantar fascia}

Hicks described a windlass mechanism of action for the plantar fascia. Dorsiflexion of the toes leads to a shortened effective length of the plantar fascia causing a raising of the arch. Toe extension leads to increased arch tension with the metatarsophalangeal joint as the pivot or tethering point. Hicks [5] demonstrated this definitively with cadaveric models.

When a plantar fasciotomy was performed, the cadaver would lose this windlass mechanism, thus diminishing the arch stability subsequently disallowing a more stable terminal stance and toe standing. In addition, Hicks [5] found 
Fig. 1 From Buchbinder [4]. Used with permission from NEJM. Plantar and medial views of the foot demonstrating the origin and insertion of the plantar fascia and the location of nerves in proximity to the heel. The windlass mechanism, or bowstring effect, of the plantar fascia refers to its function in raising the arch of the foot during the push-off phase of walking

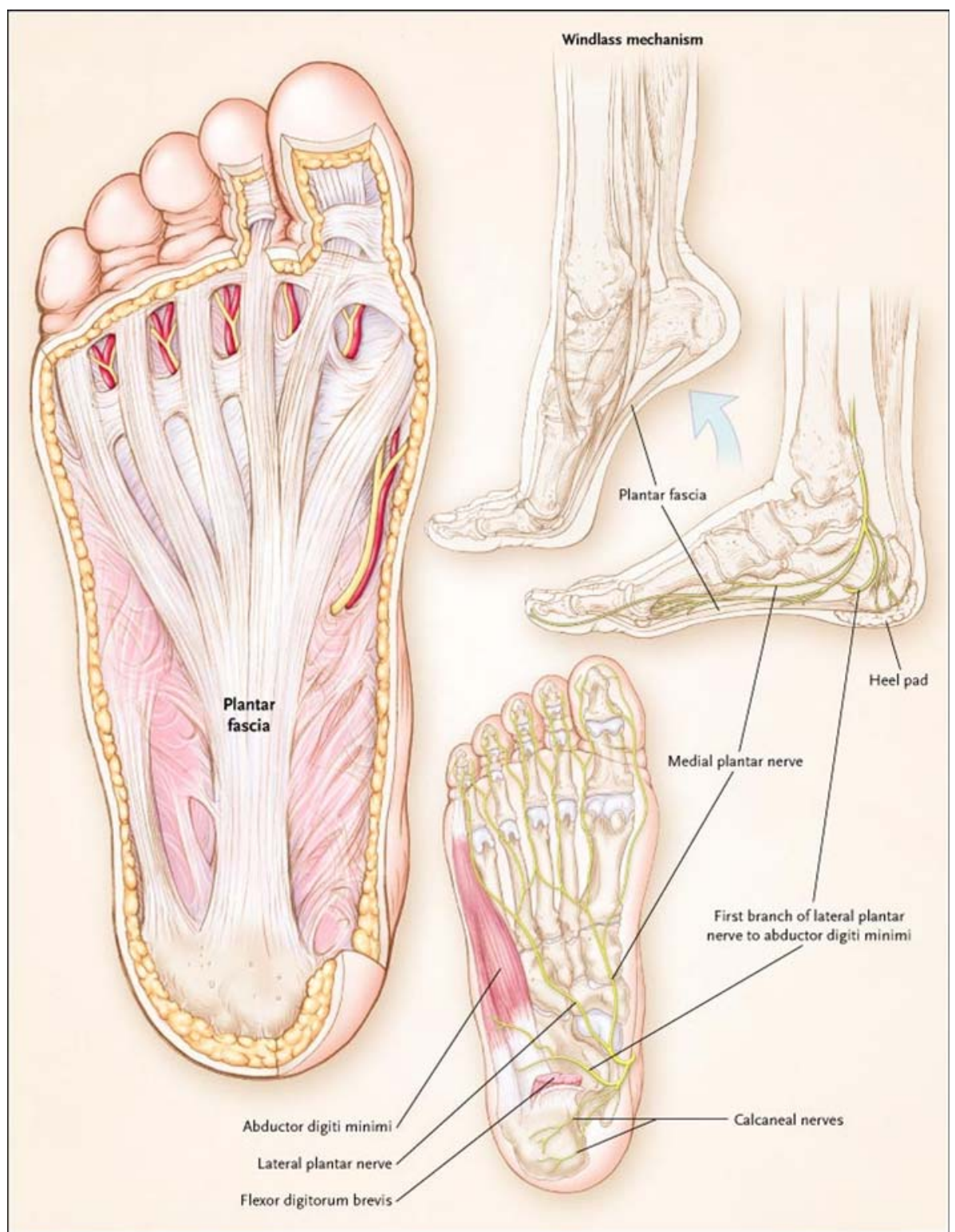

that the breaking strain for this windlass ranged from 1.4 to 3.4 times the subject's body weight. Thus, the plantar fascia, while playing a significant role in walking stability, can readily break with a minimal increase in strain.

While the exact etiology of plantar fasciitis remains unclear, it is theorized that overloading the plantar foot muscles originating at the volar calcaneus (adductor hallucis, quadratus plantae, flexor digitorum brevis, and abductor digiti minimi quinti) can lead to inflammation and consequent pain in the plantar fascia [6]. Hence, therapy for plantar fasciitis focuses on either reducing the muscle tightness that causes the initial injury or reducing the inflammation that worsens the injury.

\section{Diagnosis}

Plantar fasciitis is a clinical diagnosis. It is based on patient history and physical exam. Patients can present with local point tenderness along the medial tuberosity of the os calcis, pain on weight bearing and/or pain on first steps. Plantar fascia pain is especially evident upon dorsiflexion of the patients pedal phalanges, which further stretches the plantar fascia or windlass mechanism. Therefore, any activity that would increase stretch of the plantar fascia, such as walking barefoot without any arch support, climbing stairs, or toe walking, can worsen the pain $[4,7]$. 


\section{Method}

A Medline search from 1996 to the present was performed. Trials were included if they were randomized studies and evaluated orthoses, stretching, extracorporeal shockwave, BTX-A, or corticosteroid injection as therapies for plantar fasciitis. Trials were excluded if they did not meet the above criteria or if they compared the efficacy of one mode of therapy to another, that is, stretching to orthoses or BTX-A to steroid therapy in plantar fasciitis. According to SORT grading of quality of evidence assessments [8], all studies used were Level 1. Case studies, retrospective studies, descriptive articles, and quasi-randomized studies were excluded from the review; however, these studies were included in the introduction and discussion sections (Table 1).

Numerous treatment measures have been used for plantar fasciitis with varied clinical benefits. Nonsurgical techniques include orthoses [9, 10], stretching [11-13], splinting, taping, topical medications with or without iontophoresis, oral nonsteroidal anti-inflammatory medications [22], extracorporeal shockwave therapy [14-18], laser, and percutaneous injections with steroid $[20,21]$ or botulinum toxin type A. Surgical options with endoscopic or open fasciotomy can be the last resort for patients with intractable plantar fasciitis [23], but are not discussed further in the article.

\section{Orthoses}

Landorf et al. described a participant blinded randomized trial where they compared the response of 135 patients with plantar fasciitis to soft thin foam sham orthoses, prefabricated firm foam orthoses, and customized semi-rigid plastic orthoses over 3 months. While they were able to show statistically significant results for improved function with the prefabricated and customized orthoses as compared to the sham, Landorf et al. [9] were unable to show statistical significance for improvements in pain and concluded that both customized and prefabricated orthoses were efficacious for the treatment of plantar fasciitis.

Winemiller et al. compared the response of 101 patients to active bipolar magnet cushioned insoles or sham insoles over 8 weeks. They found that there was no statistically significant difference in improvement in subjective heel pain between different insole modes; however, there was a statistically significant improvement in both the magnetic and nonmagnetic groups in morning foot pain intensity. Winemiller et al. [10] claimed that

Table 1 SORT graded as per Ebell et al. [8]

\begin{tabular}{|c|c|c|c|c|c|c|}
\hline Subject & Authors & $\begin{array}{l}\text { Symptom } \\
\text { duration } \\
\text { prior to therapy }\end{array}$ & $\begin{array}{l}\text { Pain free time } \\
\text { after therapy }\end{array}$ & $\begin{array}{l}\text { Significant } \\
\text { improvement } \\
\text { from baseline? }\end{array}$ & $\begin{array}{l}\text { Therapy } \\
\text { grade }\end{array}$ & Consistency \\
\hline \multirow[t]{3}{*}{ Orthoses } & & & & & A & Consistent \\
\hline & Landorf et al. [9] & 4 weeks & 3 months & Yes & & \\
\hline & Winemiller et al. [10] & 30 days & $\begin{array}{l}4 \text { weeks, } \\
8 \text { weeks }\end{array}$ & Yes & & \\
\hline \multirow[t]{4}{*}{ Stretching } & & & & & B & Consistent \\
\hline & DiGiovanni et al. [11] & 10 months & 8 weeks & Yes & & \\
\hline & DiGiovanni et al. [12] & 10 months & 2 years & Yes & & \\
\hline & Radford et al. [13] & 4 weeks & 2 weeks & No & & \\
\hline \multicolumn{3}{|c|}{ Extracorporeal shockwave therapy } & & & A & Inconsistent \\
\hline & Haake et al. [14] & 6 months & 12 weeks, 1 year & No & & \\
\hline & Speed et al. [15] & 3 months & 6 months & No & & \\
\hline & Buchbinder et al. [16] & 6 weeks & 12 weeks & No & & \\
\hline & Kudo et al. [17] & 6 months & 3 months & Yes & & \\
\hline & Ogden et al. [18] & N/A & 3 months, 1 year & Yes & & \\
\hline \multirow[t]{2}{*}{ BTX-A } & & & & & B & Consistent \\
\hline & Babcock et al. [19] & 6 months & $\begin{array}{l}3 \text { weeks, } \\
8 \text { weeks }\end{array}$ & Yes & & \\
\hline \multicolumn{3}{|c|}{ Steroid injection/iontophoresis } & & & A & Consistent \\
\hline & Crawford et al. [20] & N/A & 1 month & Yes & & \\
\hline & Gudeman et al. [21] & N/A & 2 weeks & Yes & & \\
\hline
\end{tabular}

Note: All are Level 1 quality studies 
magnetic insoles provide no additional benefit as compared to nonmagnetic.

\section{Stretch}

DiGiovanni et al. assessed the role of Achilles tendon stretching versus plantar fascia stretching in a randomized study of 101 patients. Both Achilles stretching groups and plantar fascia stretching groups appreciated a decrease in pain upon first steps in the morning as well as increased function; however, the plantar stretchers appreciated a statistically significant improvement in activity function and first step pain as compared to the Achilles stretchers [11].

DiGiovanni further assessed the improvement of plantar fascia versus Achilles tendon stretching by directly comparing the two in a crossover study of the same patient pool with a 2-year follow up. DiGiovanni et al. switched Achilles tendon group to plantar fascia stretching after the initial 8 weeks of the trial. After 2 years, the Achilles tendon patient pool was reassessed for maximum pain level and pain on first steps in the morning. While there was a significant difference between the two groups at 8 weeks, there was no statistical difference between the two at 2 years, with further improvement in pain in both groups [12].

Radford recruited 92 subjects for their study that compared calf muscle stretching with sham ultrasound to sham ultrasound alone. The study tested the short-term effectiveness of stretching. In the 14 days that the study lasted, there was no statistically significant difference between stretch and sham groups. Radford et al. concluded that short-term stretching shows no statistically significant benefit for plantar fasciitis [13].

\section{Extracorporeal shockwave therapy}

Haake et al. assessed the efficacy of extracorporeal shockwave therapy (ESWT) in 272 subjects. The patients employed in this study had tried more conservative methods of plantar fasciitis therapy without effect for approximately 6 months. The study pool either received ESWT or sham therapy. The patients were assessed after 12 weeks. Haake et al. found that approximately threefourth of their study pool improved regardless of sham or ESWT. Consequently, they showed that ESWT was "ineffective in the treatment of chronic plantar fasciitis" [14].

Speed studied 88 patients who had plantar fasciitis for at least 3 months each. The patients were exposed to therapeutic sessions with sham or ESWT once every month for a total of 3 months. Three effects were assessed: day pain, night pain, and "morning startup pain." The patients were assessed at the beginning of the study, the beginning of the treatment, and at 1 and 3 months after completion of the treatment. Over the 6-month course of the study, no statistical significance was shown between sham and ESWT groups in the above categories [15].

Buchbinder et al. recruited 166 patients for their study in which they used ultrasound guided ESWT on patients who had plantar fasciitis for at least 6 weeks. Statistically significant improvements were appreciated in both the placebo/sham groups and ESWT groups in morning step pain, general activity pain, and overall pain characterization. Buchbinder et al. [16] demonstrated that ESWT provided no greater benefit than placebo.

Kudo et al. performed a confirmatory study in which they compared ESWT to sham ESWT in 114 patients with plantar fasciitis for at least 6 months and resistant to other therapies. Patients were excluded from the study if they had used other modes of therapy within 2 weeks of their treatment. The patients were exposed to a single treatment. This study demonstrated a statistically significant improvement in first steps pain by visual analog scale at 3 months. Kudo et al. [17] reported that ESWT provides for a beneficial treatment in recalcitrant plantar fasciitis.

Ogden et al. found ESWT to be an effective treatment for plantar fasciitis in their two phase trial. In the first phase, they formulated and finalized their protocol with 20 nonblinded patients. In the phase two trial, Ogden randomized 293 patients to receive either ESWT and an ankle block or a mild anesthetic and nontransmitted shockwaves. They found significant differences in investigator assessment of heel pain at 4,8 , and 12 weeks and at 12 months using a dolorimeter-based visual analog scale. Significance was also found in subjective self-assessment of morning heel pain at the same time intervals. However, in selfassessment of activity related pain, significance could be found at 4, 8, and 12 weeks, but not at 12 months. Hence, Ogden et al. [18] were able to complete that ESWT shows beneficial response in plantar fasciitis up to and for at least 1 year.

\section{Botulinum toxin type A (BTX-A)}

Babcock et al. investigated 27 patients (43 plantar fasciae) with duration of symptoms for at least 6 months. Either BTX-A was injected into the feet of the therapy group or saline into the sham group. In the case of patients with bilateral plantar fasciitis, saline was injected into one foot and BTX-A in the other. The authors showed that the BTXA groups had statistically significant improvements at 3 and 8 weeks in all variables tested (Maryland foot score, pain visual analog scale, pressure algometry response, and pain relief visual analog scale). Babcock et al. [19] 
finalized that BTX-A injections into the plantar fascia improves the patients pain level.

\section{Steroid}

Crawford et al. described 106 patients, which they divided into four groups: prednisolone with lignocaine, prednisolone with lignocaine after a tibial block, lignocaine alone, or lignocaine after a tibial block. There was no statistically significant difference in pain levels when prednisolone was given with tibial block or when anesthetic was given. There was a statistically significant decrease in pain levels according to visual analog scales in both groups of patients receiving steroid after 1 month; however, after 3 months there was no statistical significant difference. Hence, Crawford et al. [20] concluded that steroid injections can provide short-term relief.

Gudeman assessed the efficacy of steroid iontophoresis in 40 feet ( 37 subjects). Feet were divided equally into a group that received phosphate buffered solution iontophoresis and one that received dexamethasone iontophoresis. The treatments lasted for 2 weeks during which each foot received six iontophereses. The Maryland foot score was used to analyze the groups. The active group showed significantly greater improvement immediately after treatment. However, both groups showed positive results at 1 month follow up. Ultimately, Gudeman et al. [21] found that dexamethasone iontophoresis is effective in improving pain immediately after treatment, but not more than placebo in the longer term.

\section{Discussion}

As evidenced above, multiple modes of therapy can lead to pain reduction in plantar fasciitis. Initial conservative management provides satisfactory results in approximately $80-90 \%$ of cases in clinical practice [24-27]. When more conservative management is unsuccessful, steroid injection is a preferred option [28-30]. Although steroid injection is the mainstay for the management of many hyper inflammatory disorders, there is little known about steroid affect at the cellular level and, consequently, little about the etiology of the risks of connective tissue rupture after the same [31].

Fascial rupture, which can be likened to tendon rupture, was investigated by Kennedy and Willis, who found that failing strengths decreased by $35 \%$ after administration of steroid in vitro to Achilles tendons. Cystic spaces and collagen necrosis were appreciated in their steroid group. This result continued through to 7 days. However, at 2 and 4 weeks following injection, these cystic spaces were replaced by an eosinophillic staining material and fibroblast proliferation was noted. Disordered collagen deposition was appreciated under scanning electron microscope. In addition, the failing strength returned to that of the control subjects. By 6 weeks, full biomechanical integrity was reestablished as evidenced by reorganization of collagen into parallel fibers. Hence, Kennedy and Willis concluded that physiologic dosed steroid injection weakens normal tendons for up to 14 days through collagen necrosis. They also recommend limited physical activity for the 2 weeks following injection and against repeated injection [24].

Furthermore, Wong et al. [32] showed that glucocorticoids suppress cellular metabolism and collagen synthesis in cultured human tenocytes and proteoglycan production [31]. Proteoglycan synthesis has a role in extracellular matrix and collagen matrix fibrillinogenesis. Wong et al. [31, 33] concluded that spontaneous rupture following steroid injection may occur through suppression of proteoglycan synthesis.

While steroid injection and iontophoresis can significantly improve foot pain in plantar fasciitis, the two methods warrant further investigation. As evidenced by Crawford and Gudeman, steroid therapy in plantar fasciitis plays a significant role in short-term therapy [20, 21]. However, a number of complications were noted including plantar fascial rupture, plantar fat pad atrophy, lateral plantar nerve injury secondary to injection, and calcaneal osteomyelitis and in iontophoresis, burning of the underlying skin [21, 34-39].

Fascial rupture and fat pad atrophy are especially serious complications as they can lead to intractable complications. Fascial rupture interrupts the intrinsic windlass mechanism of the foot and can promote further inflammation in the surrounding tissue, thus promoting pain. In addition, plantar fat pad atrophy diminishes subcalcaneal cushioning, availing the plantar fascia to further insult and, hence, more pain. Acevedo et al. appreciated symptomatic rupture in approximately $10 \%$ of subjects. Their retrospective chart review showed that approximately two-thirds of the patients (29/44) developed sudden onset rupture with a subsequent inability to weight bear, whereas one-third did not have acute onset. All of the ruptured patients presented with new additional symptoms including long arch pain, lateral mid foot pain, and an exam showing decreased windlass tension [35].

Treatment of plantar fasciitis rupture remains unclear. Treatment options for the rupture are open surgical repair, endoscopic repair, and nonoperative measures. Sellman and Acevedo et al. demonstrated that $50 \%$ of symptomatic ruptured plantar fasciitis patients have long-term complications [36]. Acevedo and Beskin [35] used a special treatment protocol including a "boot brace" which was beneficial for expedited symptom improvement with a switch to a stiff soled athletic shoe with an arch support 
orthosis. Daly et al. [40] performed plantar fasciotomy for those patients with intractable plantar fasciitis with resolution of symptomology in $57 \%$ of the patients.

Because of the recent availability and facility of ultrasound (US) to improve needle placement accuracy and confirmatory magnetic resonance imaging (MRI) in clinical practice, enhanced therapeutic response rates have been reflected in some of the studies [29, 30, 35, 41]. Tsai et al. described the objectivity of US-guided steroid injection to plantar fascia by demonstrating a decrease in fascial thickness and loss of hypoechogenicity. In addition, following single US-guided steroid injection, fat pad atrophy, which was theorized to cause worsening pedal dynamics, was not observed [30]. Heel fat pad inflammation and degeneration may cause pain and can be misdiagnosed as plantar fasciitis [42]. Further, MRI or US can be used to make appropriate diagnoses, which is particularly important for patients with chronic polyarthritis.

Nevertheless, these complications are uncommon and preventable; rupture may occur without steroid injections [35]. Paying special attention to history and physical examination change can lend some clues to associated complications. Monitoring sudden pain loss, change of patient pain location, developing numbness, changes in plantar fascial and fat pad thickness, edema, palpable masses, asymmetric flexible hammertoe deformities of the 2nd, 3rd, and 4th toes without nerve injury, and utilizing US for follow ups and injection placement can help to alleviate negative outcomes. Further, limiting the number and frequency of steroid injections and educating patients on reduction of aggressive physical activity during a 2-week post-injection period should be suggested [35].

\section{Conclusion}

Steroid therapy is a valuable adjunct to other therapy measures, including plantar fascial stretching. However, one should be cognizant that overuse of steroid injection can lead to complications. US guidance should be utilized to improve injection target and monitor soft tissue changes, thus preventing complication.

\section{References}

1. Riddle DL, Schappert SM. Volume of ambulatory care visits and patterns of care for patients diagnosed with plantar fasciitis: a national study of medical doctors. Foot Ankle Int. 2004;25(5): 303-10.

2. Wearing SC, Smeathers JE, Urry SR, Hennig EM, Hills AP. The pathomechanics of plantar fasciitis. Sports Med. 2006;36(7): 585-611.
3. Moore KL, Agur AMR, Moore KL, Agur AMR. Essential clinical anatomy. Philadelphia: Lippincott Williams \& Wilkins; 1995.

4. Buchbinder R. Clinical practice. Plantar fasciitis. N Engl J Med. 2004;350(21):2159-66.

5. Hicks JH. The mechanics of the foot. II. The plantar aponeurosis and the arch. J Anat. 1954;88(1):25-30.

6. Braddom RL, Buschbacher RM. Physical medicine \& rehabilitation, 3rd edn. Philadelphia, PA: Saunders Elsevier; 2007.

7. American College of Foot and Ankle Surgeons. The diagnosis and treatment of heel pain. J Foot Ankle Surg. 2001;40(5):329-40.

8. Ebell MH, Siwek J, Weiss BD, Woolf SH, Susman J, Ewigman B, et al. Strength of recommendation taxonomy (SORT): a patient-centered approach to grading evidence in the medical literature. Am Fam Physician. 2004;69(3):548-56.

9. Landorf KB, Keenan AM, Herbert RD. Effectiveness of foot orthoses to treat plantar fasciitis: a randomized trial. Arch Intern Med. 2006;166(12):1305-10.

10. Winemiller MH, Billow RG, Laskowski ER, Harmsen WS. Effect of magnetic vs sham-magnetic insoles on plantar heel pain: a randomized controlled trial. JAMA. 2003;290(11):1474-8.

11. DiGiovanni BF, Nawoczenski DA, Lintal ME, Moore EA, Murray JC, Wilding GE, et al. Tissue-specific plantar fasciastretching exercise enhances outcomes in patients with chronic heel pain. A prospective, randomized study. J Bone Joint Surg. 2003;85A(7):1270-7.

12. Digiovanni BF, Nawoczenski DA, Malay DP, Graci PA, Williams TT, Wilding GE, et al. Plantar fascia-specific stretching exercise improves outcomes in patients with chronic plantar fasciitis. A prospective clinical trial with two-year follow-up. J Bone Joint Surg. 2006;88(8):1775-81.

13. Radford JA, Landorf KB, Buchbinder R, Cook C. Effectiveness of calf muscle stretching for the short-term treatment of plantar heel pain: a randomised trial. BMC Musculoskelet Disord. 2007;8:36.

14. Haake M, Buch M, Schoellner C, Goebel F, Vogel M, Mueller I, et al. Extracorporeal shock wave therapy for plantar fasciitis: randomised controlled multicentre trial. BMJ. 2003;327(7406):75.

15. Speed CA, Nichols D, Wies J, Humphreys H, Richards C, Burnet $\mathrm{S}$, et al. Extracorporeal shock wave therapy for plantar fasciitis. A double blind randomised controlled trial. J Orthop Res. 2003; 21(5):937-40.

16. Buchbinder R, Ptasznik R, Gordon J, Buchanan J, Prabaharan V, Forbes A. Ultrasound-guided extracorporeal shock wave therapy for plantar fasciitis: a randomized controlled trial. JAMA. 2002;288(11):1364-72.

17. Kudo P, Dainty K, Clarfield M, Coughlin L, Lavoie P, Lebrun C. Randomized, placebo-controlled, double-blind clinical trial evaluating the treatment of plantar fasciitis with an extracoporeal shockwave therapy (ESWT) device: a North American confirmatory study. J Orthop Res. 2006;24(2):115-23.

18. Ogden JA, Alvarez RG, Levitt RL, Johnson JE, Marlow ME. Electrohydraulic high-energy shock-wave treatment for chronic plantar fasciitis. J Bone Joint Surg. 2004;86A(10):2216-28.

19. Babcock MS, Foster L, Pasquina P, Jabbari B. Treatment of pain attributed to plantar fasciitis with botulinum toxin a: a short-term, randomized, placebo-controlled, double-blind study. Am J Phys Med Rehabil. 2005;84(9):649-54.

20. Crawford F, Atkins D, Young P, Edwards J. Steroid injection for heel pain: evidence of short-term effectiveness. A randomized controlled trial. Rheumatology (Oxford, England). 1999;38(10): 974-7.

21. Gudeman SD, Eisele SA, Heidt RS Jr, Colosimo AJ, Stroupe AL. Treatment of plantar fasciitis by iontophoresis of $0.4 \%$ dexamethasone. A randomized, double-blind, placebo-controlled study. Am J Sports Med. 1997;25(3):312-6. 
22. Donley BG, Moore T, Sferra J, Gozdanovic J, Smith R. The efficacy of oral nonsteroidal anti-inflammatory medication (NSAID) in the treatment of plantar fasciitis: a randomized, prospective, placebo-controlled study. Foot Ankle Int. 2007; 28(1):20-3.

23. Kinley S, Frascone S, Calderone D, Wertheimer SJ, Squire MA, Wiseman FA. Endoscopic plantar fasciotomy versus traditional heel spur surgery: a prospective study. J Foot Ankle Surg. 1993;32(6):595-603.

24. Kennedy JC, Willis RB. The effects of local steroid injections on tendons: a biomechanical and microscopic correlative study. Am J Sports Med. 1976;4(1):11-21.

25. Wolgin M, Cook C, Graham C, Mauldin D. Conservative treatment of plantar heel pain: long-term follow-up. Foot Ankle Int. 1994;15(3):97-102.

26. Schepsis AA, Leach RE, Gorzyca J. Plantar fasciitis. Etiology, treatment, surgical results, and review of the literature. Clin Orthop Relat Res. 1991;266:185-96.

27. Gill LH. Plantar fasciitis: diagnosis and conservative management. J Am Acad Orthop Surg. 1997;5(2):109-17.

28. Bordelon RL. Subcalcaneal pain. A method of evaluation and plan for treatment. Clin Orthop Relat Res. 1983;177:49-53.

29. Kane D, Greaney T, Bresnihan B, Gibney R, FitzGerald O. Ultrasound guided injection of recalcitrant plantar fasciitis. Ann Rheum Dis. 1998;57(6):383-4.

30. Tsai WC, Wang CL, Tang FT, Hsu TC, Hsu KH, Wong MK. Treatment of proximal plantar fasciitis with ultrasound-guided steroid injection. Arch Phys Med Rehabil. 2000;81(10):1416-21.

31. Wong MW, Tang YY, Lee SK, Fu BS. Glucocorticoids suppress proteoglycan production by human tenocytes. Acta Orthop. 2005;76(6):927-31.
32. Wong MW, Tang YN, Fu SC, Lee KM, Chan KM. Triamcinolone suppresses human tenocyte cellular activity and collagen synthesis. Clin Orthop Relat Res. 2004;421:277-81.

33. Scott JE, Hughes EW. Proteoglycan-collagen relationships in developing chick and bovine tendons. Influence of the physiological environment. Connect Tissue Res. 1986;14(4):267-78.

34. DeMaio M, Paine R, Mangine RE, Drez D Jr. Plantar fasciitis. Orthopedics. 1993;16(10):1153-63.

35. Acevedo JI, Beskin JL. Complications of plantar fascia rupture associated with corticosteroid injection. Foot Ankle Int. 1998; 19(2):91-7.

36. Sellman JR. Plantar fascia rupture associated with corticosteroid injection. Foot Ankle Int. 1994;15(7):376-81.

37. Leach R, Jones R, Silva T. Rupture of the plantar fascia in athletes. J Bone Joint Surg. 1978;60(4):537-9.

38. Snow DM, Reading J, Dalal R. Lateral plantar nerve injury following steroid injection for plantar fasciitis. Br J Sports Med. 2005;39(12):e41; discussion e41.

39. Gidumal R, Evanski P. Calcaneal osteomyelitis following steroid injection: a case report. Foot Ankle. 1985;6(1):44-6.

40. Daly PJ, Kitaoka HB, Chao EY. Plantar fasciotomy for intractable plantar fasciitis: clinical results and biomechanical evaluation. Foot Ankle. 1992;13(4):188-95.

41. Genc H, Saracoglu M, Nacir B, Erdem HR, Kacar M. Long-term ultrasonographic follow-up of plantar fasciitis patients treated with steroid injection. Joint Bone Spine. 2005;72(1):61-5.

42. Falsetti P, Frediani B, Acciai C, Baldi F, Filippou G, Marcolongo R. Heel fat pad involvement in rheumatoid arthritis and in spondyloarthropathies: an ultrasonographic study. Scand J Rheumatol. 2004;33(5):327-31. 